\title{
Caracterización nutricional de filetes de bagre yaque (Leiarius marmoratus) procedentes de extracción natural y cultivo
}

\author{
Nutritional characterisation of filetes of bagre yaque \\ (Leiarius marmoratus) pertinent of natural extraction and crop
}

Caracterização nutricional de filetes de bagre yaque (Leiarius marmoratus) procedente de extração natural e cultivo

\author{
Nubia E. Cruz-Casallas ${ }^{1}$, Víctor M. Medina-Robles ${ }^{2 *}$, Pablo E. Cruz-Casallas ${ }^{3 *}$, \\ Héctor Suárez-Mahecha ${ }^{4}$
}

Ingeniera Agrónoma, Esp, cMSc; ${ }^{2} \mathrm{MVZ}, \mathrm{MSc} ;{ }^{3} \mathrm{MVZ}, \mathrm{MSc}, \mathrm{PhD}$

4 MVZ, PhD, Instituto de Ciencia y Tecnología de Alimentos ICTA. Universidad Nacional de Colombia. Sede Bogotá

* Grupo de Investigación sobre Reproducción y Toxicología de Organismos Acuáticos (GRITOX). Instituto de Acuicultura, Universidad de los Llanos, Villavicencio, Colombia.

Email: nuescruz@yahoo.com.ar

Recibido: septiembre 4 de $2012 \quad$ Aceptado: noviembre 29 de 2012

\begin{abstract}
Resumen
La introducción de especies nativas a los sistemas acuícolas requiere del conocimiento de su valor nutricional bajo las condiciones particulares de manejo utilizadas. El objetivo de este trabajo fue determinar la composición proximal y el perfil de ácidos grasos de filetes de bagre Yaque (Leiarius marmoratus) procedentes de extracción natural (EN) y de cultivo, almacenados durante 120 días bajo condiciones de congelación $\left(-18^{\circ} \mathrm{C}\right)$. Los filetes de $\mathrm{EN}$ presentaron alto contenido de proteína $(19,8 \pm 0,2 \%)$ y de lípidos $(4,6 \pm 0,3 \%)$; de igual forma, presentaron $47,0 \pm 0,3 \%$ de ácidos grasos saturados (SFA),

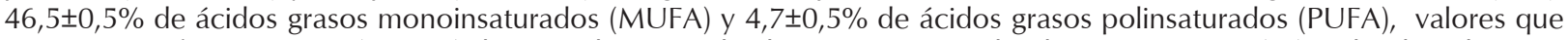
variaron significativamente $(p<0,05)$ durante el tiempo de almacenamiento. El índice aterogénico (IA) y el índice de trombogenicidad (IT) fueron de 1,6 $\pm 0,02$ y $0,9 \pm 0,01$, respectivamente. Por otra parte, los filetes procedentes de animales de cultivo presentaron alto contenido de proteína $(20,0 \pm 1,1 \%)$ y bajo contenido de lípidos $(1,5 \pm 0,3 \%)$, con $39,5 \pm 0,5 \%$ de

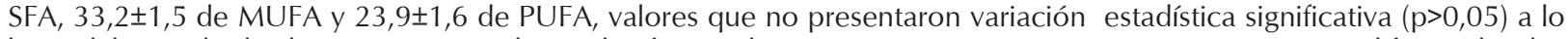
largo del periodo de almacenamiento. El IA y el IT fueron de 1,3 $\pm 0,02$ y $0,7 \pm 0,03$, respectivamente. De igual forma, las dos procedencias presentaron importantes contenidos de EPA y DHA. Con base en los anteriores resultados puede inferirse que los filetes de Yaque son de buena calidad nutricional, si se comparan con la de otros bagres comerciales.
\end{abstract}

Palabras clave: almacenamiento, composición proximal, perfil lipídico, índice aterogénico, índice de trombogenicidad.

Key words: storage, proximate composition, lipid profile, atherogenic index, index of thrombogenicity. 